\title{
Expression of IRAK-3 is associated with colitis-associated tumorigenesis in mice
}

\author{
DINGTING XU* , HANYUN ZHANG ${ }^{*}$, XIAOYING WANG and YAN CHEN \\ Department of Gastroenterology, The Second Affiliated Hospital, School of Medicine, \\ Zhejiang University, Hangzhou, Zhejiang 310009, P.R. China
}

Received July 1, 2016; Accepted May 2, 2017

DOI: $10.3892 / \mathrm{mmr} .2017 .6958$

\begin{abstract}
Interleukin-1 receptor-associated kinase-3 (IRAK-3) is a negative regulator in Toll-like receptor (TLR) pathways. The present study investigated the importance of IRAK-3 in a mouse model of chemically-induced colitis-associated tumorigenesis. The colitis-associated tumorigenesis was induced in ICR mice by the administration of 1,2-dimethyl hydrazine (DMH) and dextran sodium sulfate (DSS), termed the DMH + DSS group. In the DSS group, mice were administered with DSS; in the DMH group, mice were injected with DMH; in the control group, mice were injected with physiological saline. The clinical signs were examined for 20 weeks; tissue samples were analyzed at week 4, 9, 13 and 20. At week 20, the levels of IRAK-3 were analyzed using immunohistochemistry, western blot analysis, reverse transcription-quantitative polymerase chain reaction (RT-qPCR) analysis, and methylation-specific PCR. At week 20, the DMH + DSS group mice exhibited a decrease in total body weight and had developed canalicular adenoma or adenocarcinoma. The mice in the DSS group and DMH group presented with significant colitis at week 20. The mice with colitis-associated tumorigenesis were found to have decreased levels of IRAK-3, compared with the mice in the other groups, as evidenced by the results of the immunohistochemistry $(\mathrm{P}=0.002)$, RT-qPCR analysis $(\mathrm{P}<0.001)$ and western blot analysis $(\mathrm{P}<0.001)$. IRAK-3 methylation was observed in all experimental groups. Taken together, DMH + DSS induction in colitis led to increased inflammation and risk of tumorigenesis. IRAK-3 methylation may be a predictive factor in the transition from colitis to cancer.
\end{abstract}

Correspondence to: Dr Yan Chen,Department of Gastroenterology, The Second Affiliated Hospital, School of Medicine, Zhejiang University, 88 Jiefang Road, Hangzhou, Zhejiang 310009, P.R. China E-mail: chenyan72_72@zju.edu.cn

${ }^{*}$ Contributed equally

Key words: interleukin-1 receptor-associated kinase 3, ulcerative colitis, DNA methylation, mouse model

\section{Introduction}

Ulcerative colitis (UC) is a chronic condition characterized by continuous inflammation of the colonic mucosa and submucosa, and it is associated with an increased risk of colon, rectal and bowel cancer. A previous study reported that the worldwide incidence of UC was 1.2-20.3 cases per 100,000 individuals per year, with a prevalence of 7.6-246.0 cases per 100,000 individuals per year (1). UC is one of two conditions referred to as inflammatory bowel disease, the other condition being Crohn's disease.

Several animal models have been developed to investigate the pathogenesis of UC in detail. UC can be readily induced in animal models either chemically or by bacterial infection. The majority of animal models of UC involve mice; however, UC has previously also been induced in other animals, including zebrafish, Drosophila and pigs (2). The administration of dextran sulfate sodium, intrarectal administration of oxalozone or acetic acid, or infection with Salmonella typhimurium or Escherichia coli have all been successfully used to induce UC-like symptoms and immune responses in animal models. The interleukin (IL)-7 gene is a candidate risk gene associated with UC. T-cell receptor $\alpha$ chain (TCR $\alpha$ )-knockout mice have been reported to spontaneously develop chronic colitis, which was mediated by a Th2-type immune response closely resembling human UC, with an inflammatory pattern restricted primarily to the colonic mucosa. Thus, genetic approaches, including the knockout of IL-7 and TCR $\alpha$ genes have also been used to induce chronic colitis in animal models (3).

There is substantial evidence suggesting that UC-associated colon cancer is responsible for $10-15 \%$ of UC-associated mortality (4). However, how the chronic inflammation of UC develops into cancer remains to be elucidated.

Toll-like receptor (TLR) and innate immune processes have been shown to be involved in the pathogenesis of colitis-associated tumorigenesis (5). The expression of TLR4 has been found to be increased in colitis-associated tumorigenesis and is associated with the degree of dysplasia (2). IL-1 receptor-associated kinase (IRAK) and MyD88 are key factors involved in TLR4-associated signal transduction. IRAK-1 and IRAK-4 are the active members of the IRAK family, whereas IRAK-2 and IRAK-3, also known as IRAK-M, are the inactive members of this family. The expression of IRAK-3 is localized to the monocyte and macrophage populations only, and it prevents 
the formation of a complex containing IRAK-1 and IRAK-4. The other members of the IRAK family are ubiquitous. Studies have reported increased inflammation in IRAK- $3^{-/}$cells and mice, suggesting that IRAK-3 is a negative regulator in TLR pathways, whereas the lack of IRAK-3 is known to be associated with inflammation and tumorigenesis (6-8). Specifically, IRAK-3 mediates TLR7-induced MEKK3-dependent second wave activation of nuclear factor- $\mathrm{\kappa B}(\mathrm{NF}-\mathrm{\kappa B})$ to produce inhibitory molecules as negative feedback in the pathway, and exerts an inhibitory effect on the translational control of cytokines and chemokines $(9,10)$. Previous studies have also demonstrated that chronic inflammation is frequently accompanied by methylation in the development of UC, which occurs prior to dysplasia (11). Although the exact mechanism of methylation and its association with tumorigenesis remains to be elucidated, these findings indicate that the early detection of methylation may serve to predict and consequently prevent cancer.

Therefore, the present study aimed to determine the role of IRAK-3 and changes in its methylation levels in a mouse model of chemically induced colitis-associated cancer, in order to examine the potential of IRAK-3 as a therapeutic target.

\section{Materials and methods}

Animals. Male ICR mice (5 weeks old) weighing 25-33 g were purchased from the Shanghai Laboratory Animal Research Center (Shanghai, China; certificate no. SCXK2013-0016). Mice were maintained under standard conditions with a temperature of $24 \pm 1^{\circ} \mathrm{C}$ and 12-h light/dark cycle, and were fed standard laboratory chow and tap water ad libitum. The experiment was performed following 3 days of acclimatization. All procedures were performed in strict accordance with the legislation of the P.R China. The study was approved by the animal ethics committee of the Second Affiliated Hospital, School of Medicine, Zhejiang University (Hangzhou, China).

Development of the mouse model. The ICR mice were randomly allocated into four groups ( $\mathrm{n}=8$ mice/group). The mice in the control group were injected intraperitoneally with $200 \mu 1$ physiological saline, whereas the mice in the DMH and DMH + DSS groups were injected intraperitoneally with $15 \mathrm{mg} / \mathrm{kg}$ 1,2-dimethyl hydrazine (DMH) dissolved in physiological saline (pH 6.5-7.0). After 1 week, the mice in the DSS and DMH + DSS groups were provided with 2\% dextran sodium sulfate (DSS) dissolved in tap water, which was provided ad libitum for 7 days, whereas the mice in the control and DMH groups were provided with tap water ad libitum. The body weights, levels of activity and clinical signs of the mice were monitored every week for a period of 20 weeks. At weeks 4, 9, 13 and 20, one mouse from each group was sacrificed, and tissue samples were collected for histopathological examination. At week 20, the levels of IRAK3 were analyzed using immunohistochemistry, western blot analysis, reverse transcription-quantitative polymerase chain reaction (RT-qPCR) analysis and methylation-specific PCR (MSP) analysis.

Histological examination. For histological examination, one mouse from each group was sacrificed at weeks 4,9, 13 and 20.
The abdominal cavity of the mouse was opened, and the colon was isolated and generally scored. A $1-\mathrm{cm}$ specimen was then cut at $3 \mathrm{~cm}$ from the anal verge, and placed in $40 \mathrm{~g} / \mathrm{l}$ formalin at room temperature for $48 \mathrm{~h}$. The specimens were embedded in paraffin and then cut into 4-7- $\mu$ m-thick sections. The sections were stained with $2 \mathrm{~g} / \mathrm{l}$ hematoxylin for $5 \mathrm{~min}$ and $0.5 \%$ eosin for 2-3 $\mathrm{min}$ at room temperature and examined under a light microscope.

Immunohistochemical analysis of IRAK-3. The immunohistochemical staining was performed using a standard avidin-biotin complex technique at week 20 . The fresh frozen (4- $\mu$ m-thick) sections of mice colon were mounted onto glass slides, fixed in $100 \%$ acetone for $10 \mathrm{~min}$ at $4^{\circ} \mathrm{C}$, air-dried at room temperature for $10 \mathrm{~min}$ and rehydrated in PBS. Following blocking of endogenous peroxidase activity for $20 \mathrm{~min}$ in $3 \%$ hydrogen peroxide at room temperature, the slides were incubated for 10-15 min at room temperature with normal goat serum (Nanjing Jiancheng Bioengineering Institute, Nanjing, China) for blocking. The slides were then incubated with anti-IRAK3 antibodies (cat no. ab238; 1:1,500; Abcam, Cambridge, MA, USA) at $4^{\circ} \mathrm{C}$ overnight. The slides were then kept for 20-30 min at room temperature, washed in PBS and then incubated for 10-15 min at room temperature with a goat anti-rabbit horseradish peroxidase-conjugated secondary antibody (cat no. ab6721; 1:1,000; Abcam). Following washing with PBS, the slides were incubated with streptavidin-biotin complex (cat no. ab7403; 1:15,000; Abcam) for $30 \mathrm{~min}$ at room temperature, then washed in PBS 3 times and stained with diaminobenzidine (cat no. ab103723; Abcam) for $20 \mathrm{~min}$ at room temperature. Finally, the sections were rinsed in distilled water, counterstained with $2 \mathrm{~g} / 1$ hematoxylin for $1 \mathrm{~min}$ at room temperature, washed in running tap water and mounted with mounting media. The slides were then observed under a light microscope (Olympus, Tokyo, Japan) and images were captured. Immunohistochemical data were quantified using Image-Pro Plus software version 6.0 (Media Cybernetics, Inc., Rockville, MD, USA).

$R T-q P C R$. The colon tissues were snap-frozen in liquid nitrogen, and stored at $-80^{\circ} \mathrm{C}$ until further analysis. Colon tissues were homogenized in diethyl pyrocarbonate-treated water, ice-cold PBS, 70\% ethanol and isopropyl alcohol, and total RNA was extracted using TRIzol ${ }^{\circledR}$ reagent (Invitrogen; Thermo Fisher Scientific, Inc., Waltham, MA, USA). Total RNA was reverse-transcribed into cDNA using RevertAid First Strand cDNA Synthesis (Thermo Fisher Scientific, Inc.). qPCR was performed on cDNA using a Toyobo Revertra Ace qRCR RT kit (Toyobo Co., Ltd., Osaka, Japan) and SYBR Green Real-Time PCR Master Mix (Thermo Fisher Scientific, Inc.). The qPCR reaction consisted of $25.0 \mu 1$ SYBR Green Real-Time PCR Master Mix, 5.0 $\mu \mathrm{l}$ cDNA, $16.0 \mu 1$ nuclease-free water, and $4.0 \mu \mathrm{l}$ of primer pairs, all in a total volume of $50 \mu \mathrm{l}$. The thermocycling conditions were as follows: Initial denaturation at $95^{\circ} \mathrm{C}$ for $10 \mathrm{~min}$, followed by 40 cycles at $95^{\circ} \mathrm{C}$ for $15 \mathrm{sec}$, at $60^{\circ} \mathrm{C}$ for $30 \mathrm{sec}$ and at $72^{\circ} \mathrm{C}$ for $30 \mathrm{sec}$. The mRNA expression levels were normalized to GAPDH, which was used as the endogenous control. The following primer sequences were used: IRAK-3, forward 5'-TTGGTCCTGGGCACAGAA A-3', reverse 5'-AATAGCTCGACGATGTCCCAT-3'; and 
GAPDH, forward 5'-GGTATCGTGGAAGGACTCATGAC-3' and reverse 5'-ATGCCAGTGAGCTTCCCGTTCCCGTTC AGC-3'. Target gene expression was quantified according to the comparative Cq method (12). Experiments were performed in triplicate.

Western blot analysis. Total proteins were extracted from the colon samples obtained at week 20 using ice-cold radioimmunoprecipitation assay lysis buffer containing $50 \mathrm{mM}$ Tris- $\mathrm{HCl}$ ( $\mathrm{pH} 8.0), 150 \mathrm{mM}$ sodium chloride, $1 \mathrm{mM}$ EDTA (pH 8.0), $1 \%(\mathrm{v} / \mathrm{v})$ TritonX-100, $0.1 \%(\mathrm{~m} / \mathrm{v})$ SDS and $1 \mathrm{mM}$ phenylmethylsulfonyl fluoride. Protein concentration was determined using a Bradford assay. Equal amounts of extracted protein samples $(20 \mu \mathrm{l})$ were separated by $10 \%$ SDS-PAGE and transferred onto a polyvinylidene difluoride membrane. The membrane was blocked with 5\% nonfat dried milk in PBS containing $0.1 \%$ Tween-20 for $1 \mathrm{~h}$ at room temperature and then incubated with an anti-IRAK-3 (cat no. ab238; 1:5,000; Abcam) antibody at $4{ }^{\circ} \mathrm{C}$ overnight, followed by incubation for $2 \mathrm{~h}$ at room temperature with goat anti-rabbit horseradish peroxidase-conjugated secondary antibodies [cat no. 70-GAR007; 1:10,000; Multisciences (Lianke) Biotech Co., Ltd., Hangzhou, China]. The protein bands were visualized and documented using the ChampGel ${ }^{\mathrm{TM}} 6000$ image acquisition and analysis system (Beijing Sage Creation Science Co., Ltd., Beijing, China). Blots were semi-quantified using ImageJ software version 2.1.4.7 (National Institutes of Health, Bethesda, MD, USA).

Bisulfite modification and MSP analysis. Genomic DNA was isolated from colon tissues using the TIANamp Genomic DNA kit (Tiangen Biotech Co., Ltd., Beijing, China). Briefly, $1 \mu \mathrm{g}$ genomic DNA was bisulfite-modified using an EZ DNA Methylation-Lightning ${ }^{\mathrm{TM}}$ kit (Zymo Research Corporation, Irvine, CA, USA) according to the manufacturer's protocol. The MSP was performed at $94^{\circ} \mathrm{C}$ for $5 \mathrm{~min}$, followed by 35 cycles at $94^{\circ} \mathrm{C}$ for $30 \mathrm{sec}, 55^{\circ} \mathrm{C}$ for $30 \mathrm{sec}$ and $72^{\circ} \mathrm{C}$ for $30 \mathrm{sec}$. The final extension step was performed at $72^{\circ} \mathrm{C}$ for $10 \mathrm{~min}$. The PCR reaction consisted of $1.5 \mu \mathrm{l} 10 \mathrm{X}$ Taq Buffer, $1.0 \mu \mathrm{ldNTP}$ $(20 \mathrm{mM}), 0.5 \mu \mathrm{l}$ Taq DNA polymerase (Takara Bio, Inc., Otsu, Japan), $1.0 \mu \mathrm{l}$ cDNA, $20.0 \mu \mathrm{l}$ nuclease-free water and $1.0 \mu \mathrm{l}$ primer pairs, in a total volume of $25 \mu$ l. The following MSP primer sequences were used: IRAK-3 unmethylated, forward 5'-AAGTAATTATGGATTGAAGTTTTGA-3' and reverse 5'-CAAACAAAAACAACCTAAAACATA-3', IRAK-3 methylated, forward 5'-AAGTAATTATGGATTGAAGTTTCGA-3' and reverse 5'-CAAACAAAAACAACCTAAAACGTA-3'.

Statistical analysis. Data are expressed as the mean \pm standard deviation for each group. An independent t-test was used to evaluate differences between two treatment groups. All data were processed and analyzed using SPSS version 19.0 (IBM Corp., Armonk, NY, USA). P $<0.05$ was considered to indicate a statistically significant difference.

\section{Results}

General condition and pathological evaluations. At weeks 9 and 13 following the start of the experiment, colitis was induced in the mice by injection with $15 \mathrm{mg} / \mathrm{kg}$ DMH (DMH and DMH
Table I. Quantitative results of immunohistochemistry.

\begin{tabular}{lcccc}
\hline Factor & Control & DSS & DMH & DMH+DSS \\
\hline Area (pixels) & 675,801 & 309,303 & 605,832 & 57,288 \\
IOD & $168,967.94$ & $63,390.00$ & $138,182.08$ & $9,852.63$ \\
Intensity & 0.25003 & 0.20494 & 0.22809 & 0.17198 \\
P-value & & 0.007 & 0.040 & 0.000 \\
\hline
\end{tabular}

DSS, dextran sodium sulfate; DMH, 1,2-dimethyl hydrazine; IOD, integrated optic density.

+ DSS groups) or by provision of drinking water containing $2 \%$ DSS for 7 days (DSS and DMH + DSS groups). At week 20, the mice in the DMH + DSS group had lost body weight and developed canalicular adenoma or adenocarcinoma (Fig. 1).

Immunohistochemical localization of IRAK-3 in the colon. Positive cytoplasmic and nuclear staining for IRAK-3 was found in all groups, and this positive staining was highest in the mice without DMH or DSS (Fig. 2). By contrast, positive staining for IRAK-3 was minimal in the colitis-associated cancer model. The quantitative results of the immunohistochemistry also showed that the expression of IRAK-3 in the colon was decreased significantly in the mice with induced colitis, particularly in the mice with tumorigenesis $(\mathrm{P}=0.000$; Table I). Taken together, the results of the immunohistochemistry indicated that IRAK-3 was downregulated in the colon cells of DSS- or DMH-induced colitis, particularly in the colitis-associated cancer model.

Expression levels of IRAK-3 and gene methylation in the colon. The gene expression of $I R A K-3$ in the colon was significantly lower in mice in the DMH + DSS group with colitis-associated tumorigenesis $(\mathrm{P}<0.001$; Fig. 3). The results of the western blot analysis also revealed that the protein levels of IRAK-3 in the colon were decreased in mice with induced colitis, particularly in mice with tumorigenesis (Fig. 4), which was in line with the results of the mRNA levels of IRAK-3. Methylation of the IRAK-3 gene was observed in the DSS, DMH and DMH + DSS groups. However, the methylation levels were higher in the mice in the DMH + DSS group, compared with those in the other two groups (Fig. 5).

\section{Discussion}

Patients with UC are at an increased risk of colorectal cancer (4). TLR and innate immune processes are involved in the pathogenesis of UC-associated tumorigenesis. As the role of IRAK-3 as a negative regulator in TLR pathways is well known, the present study investigated the importance of IRAK-3 in a chemically-induced model of colitis-associated tumorigenesis in mice. Colonic epithelial cells and microbes are important components of the gut barrier. Damage to colonic epithelial cells can induce inflammation and, if the inflammation is aggravated, it increases the risk of cancer (11). DMH and DSS are widely used to induce colitis in murine models $(13,14)$. Therefore, these two agents were used in the present study to establish a murine UC model. 
A

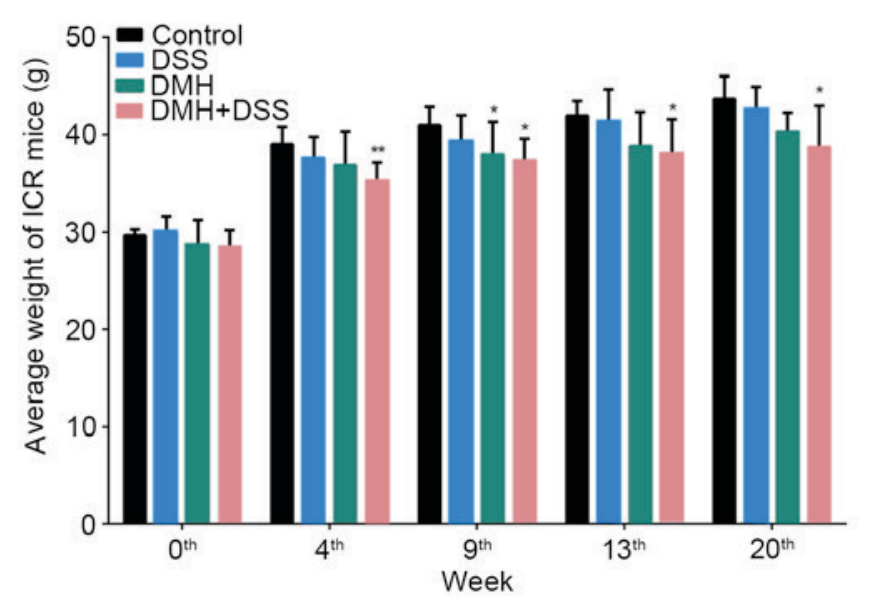

B

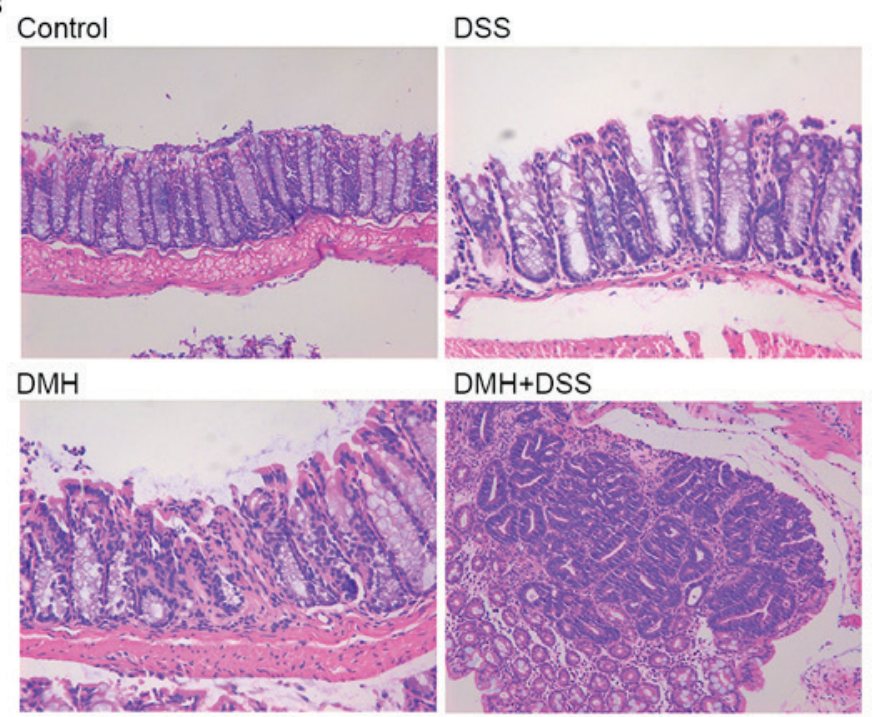

Figure 1. Comparison of body weights and histopathological analysis. (A) Graph of body weights during the experimental period. " $\mathrm{P}<0.05$ and ${ }^{* *} \mathrm{P}<0.01$, vs. control group. (B) Histopathological analyses at week 20. Mice in the DMH + DSS group developed canalicular adenoma or adenocarcinoma, and mice in the DSS and DMH groups exhibited increased colitis at week 20. Control, injected with physiological saline; DSS, provided with tap water containing DSS; DMH, injected with DMH; DMH + DSS, injected with $15 \mathrm{mg} / \mathrm{Kg}$ DMH and tap water containing DSS. Magnification, x100. DSS, dextran sodium sulfate; DMH, 1,2-dimethyl hydrazine.

Control

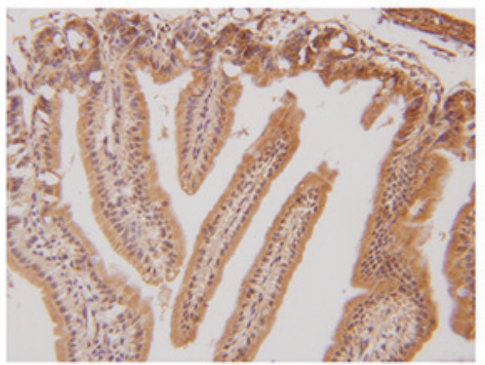

$\mathrm{DMH}$

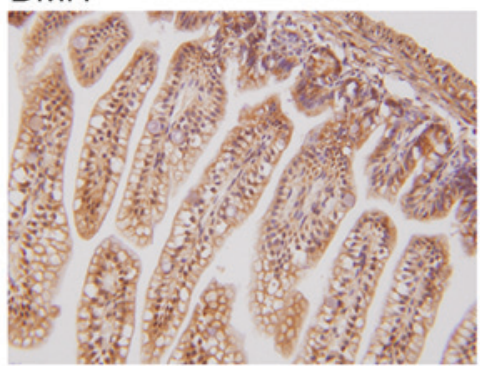

DSS

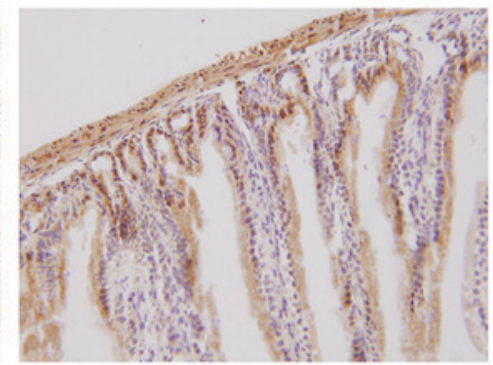

$\mathrm{DMH}+\mathrm{DSS}$

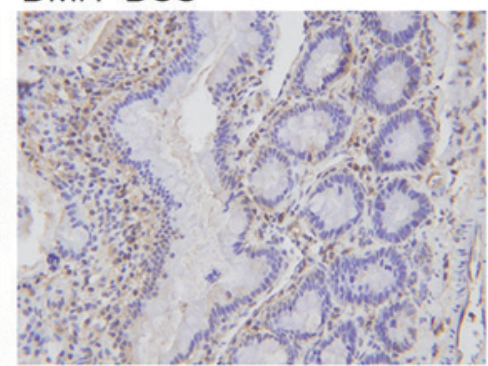

Figure 2. Immunohistochemistry of IRAK-3. Expression of IRAK-3 was decreased in the DMH + DSS group at week 20. Control, injected with physiological saline; DSS, provided with tap water containing DSS; DMH, injected with DMH; DMH + DSS, injected with $15 \mathrm{mg} / \mathrm{Kg}$ DMH and tap water containing DSS . Magnification, x200. IRAK-3, interleukin-1 receptor-associated kinase-3; DSS, dextran sodium sulfate; DMH, 1,2-dimethyl hydrazine.

The mice in the present study were administered with physiological saline, DMH, DSS, or DMH + DSS, and the expression levels of IRAK-3 were analyzed. IRAK-3 was expressed in all groups, and the positive staining for IRAK-3 was highest in the mice without DMH or DSS (Fig. 2). IRAK-3 generally acts as a negative regulator of the activation of NF- $\kappa B$, IRAK-4/IRAK-1 and IRAK-4/IRAK-2 in TLR and IL-1R signaling, and favors immunosuppression $(6-8,15)$. Jain et al (7) demonstrated that types of cancer with reduced levels of IRAK-3, but elevated levels of IRAK-1, IRAK-2, and/or IRAK-4, showed increased IRAK-4 signaling and consequently elevated levels of inflammatory molecules. The absence of IRAK-3 may further sustain IRAK-4 signaling and perpetuate a chronically inflamed tumor environment; chronic inflammation is a hallmark of tumorigenesis and tumor progression (16). Several studies have supported the conclusion that the negative regulation of IRAK-4 is important in colon cancer resistance (17). The results of the present study showed similar results in the colitis mice model. It was found that colitis and dysplasia specimens showed decreased 


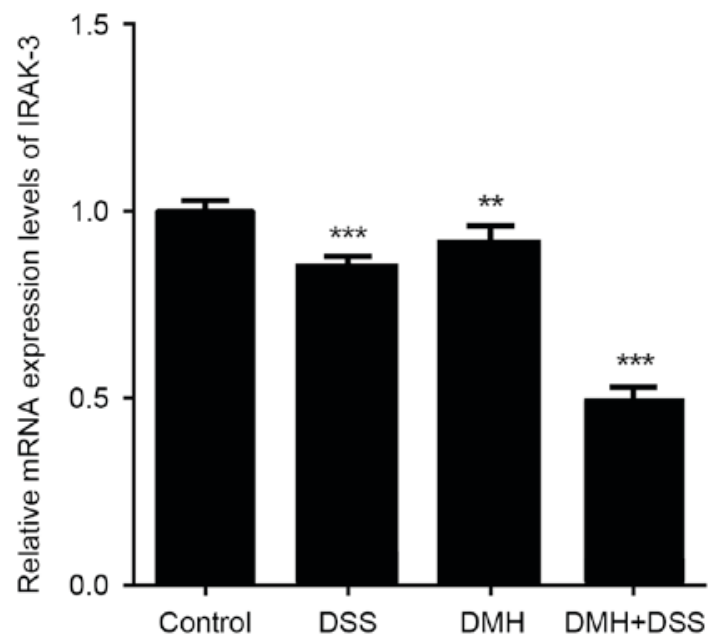

Figure 3. Reverse transcription-quantitative polymerase chain reaction analysis of IRAK-3. mRNA expression of IRAK-3 was significantly lower in the DMH + DSS group, compared with that in the other groups. ${ }^{* *} \mathrm{P}<0.01$ and ${ }^{* * * *} \mathrm{P}<0.001$, vs. control group. Control, injected with physiological saline; DSS, provided with tap water containing DSS; DMH, injected with DMH; $\mathrm{DMH}+\mathrm{DSS}$, injected with $15 \mathrm{mg} / \mathrm{Kg}$ DMH and tap water containing DSS IRAK-3, interleukin-1 receptor-associated kinase-3; DSS, dextran sodium sulfate; DMH, 1,2-dimethyl hydrazine.
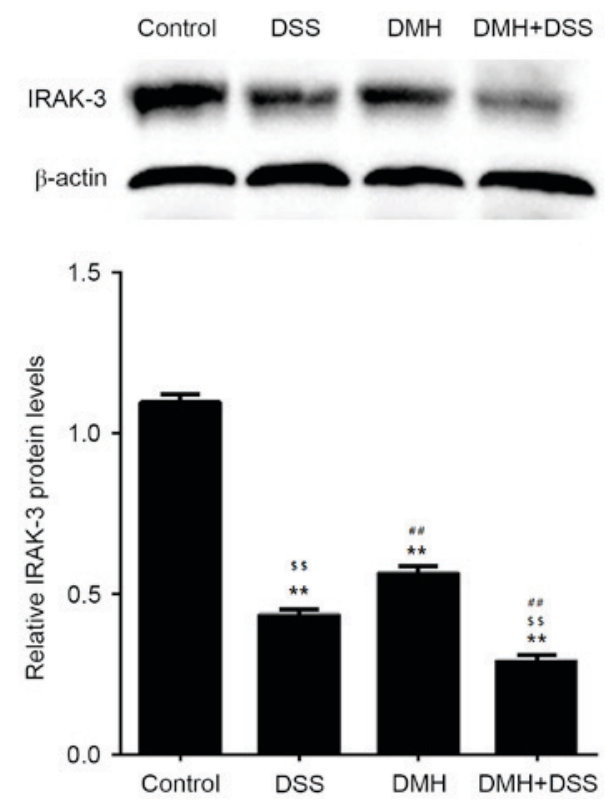

Figure 4. Western blot analysis of IRAK-3. Expression of IRAK-3 was significantly lower in the DMH + DSS group, compared with the other groups. ${ }^{* *} \mathrm{P}<0.01$ vs. Control; ${ }^{\# \#} \mathrm{P}<0.01$ vs. DSS; ${ }^{\$ \$} \mathrm{P}<0.01$ vs. DMH. Control, injected with physiological saline; DSS, provided with tap water containing DSS; DMH, injected with DMH; DMH + DSS, injected with $15 \mathrm{mg} / \mathrm{Kg}$ DMH and tap water containing DSS. IRAK-3, interleukin-1 receptor-associated kinase-3; DSS, dextran sodium sulfate; DMH, 1,2-dimethyl hydrazine.

expression of IRAK-3. However, a previous study showed that IRAK-3 may promote cancer progression by modulating macrophage activity (7). Evidence from previous studies using in vivo mouse models showed that the expression level of IRAK-3 was higher in infiltrating macrophages, and that the expression levels of IRAK-3 in the tumor cells of patients with lung cancer were significant and independent predictors of mortality rates (18-20). These data suggest that the role of

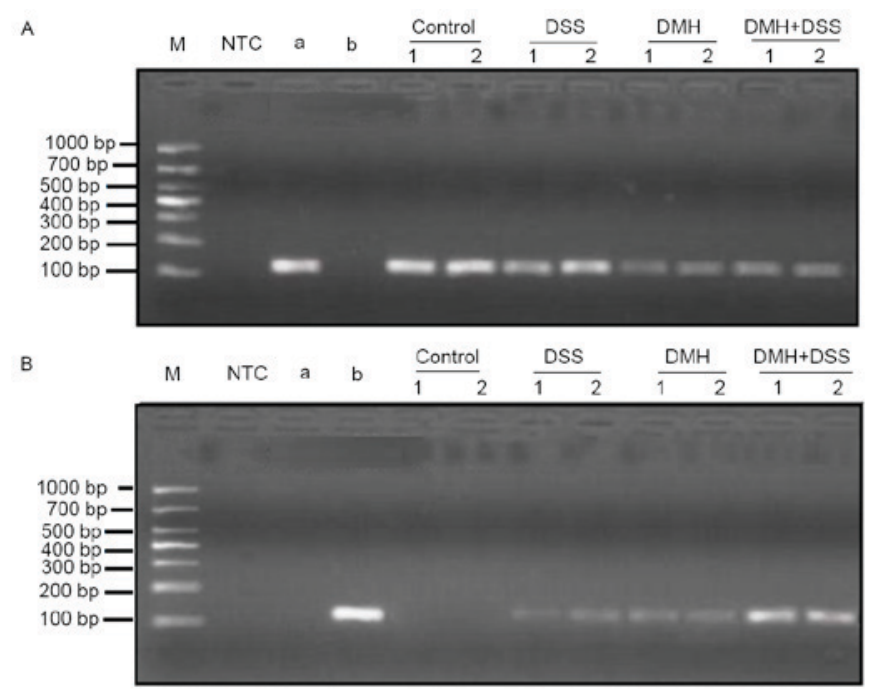

Figure 5. Gene methylation of IRAK-3. Gene methylation of IRAK-3 at week 20 was determined using methylation-specific polymerase chain reaction analysis. (A) Determination of gene expression levels of IRAK-3 in all the groups prior to treatment (unmethylated). (B) Determination of IRAK-3 gene methylation levels at week 20. Samples were examined in duplicate. NTC, no template control; a, negative reference material; b, methylation reference material; control, injected with physiological saline; DSS, provided with DSS dissolved in tap water for drinking; DMH, injected with DMH; DMH + DSS: mice were injected with $15 \mathrm{mg} / \mathrm{Kg}$ DMH and provided with DSS dissolved in tap water for drinking. IRAK03, interleukin-1 receptor-associated kinase-3; DSS, dextran sodium sulfate; DMH, 1,2-dimethyl hydrazine.

IRAK-3 remains to be fully elucidated. It may be a regulator between tumor cells and macrophages, which may prevent or promote tumorigenesis, particularly in UC. Further investigations are warranted to examine the role of IRAK-3, and its methylated form, in UC and colon cancer.

During the course of chronic inflammation, various genes undergo methylation, including, E-cadherin (21) and hyperplastic polyposis protein 1 (HPP1) (22). CpG island hypermethylation has also been reported to occur in relation to tumorigenesis or aging. A study on nonneoplastic gastric mucosa reported that chronic inflammation is closely associated with increased methylation $(23,24)$. In a previous study, the methylation of E-cadherin was found in $93 \%$ of dysplasia specimens; HPP1 was considered to be associated with the adenoma (polyp) cancer path, and methylation was found in $50 \%$ of colitis-associated cancer cases and $40 \%$ of dysplasia cases. The degree of methylation is connected with the level of inflammation (25). Ullman and Itzkowitz (11) suggested that DNA methylation occurs prior to dysplasia. These findings indicate that methylation may be a potential target for clinical intervention. The present study further investigated whether IRAK-3 is methylated, and determined the significance of this change. The results showed that IRAK-3 was methylated in the cancer chemically-induced colitis groups, supporting the hypothesis that the expression and methylation of IRAK-3 may be involved in UC and subsequent tumorigenesis. However, to examine the effect of IRAK-3 on tumorigenesis, experiments involving the knock down of the IRAK-3 gene or siRNA are required.

The present study had a number of limitations. T cells and B cells are not required for the development of 
chemically-induced colitis; therefore, the mouse models may not be an accurate reflection of human colitis. In addition, intestinal bacteria are important in the development of colitis. Further investigations should take these factors into account when establishing animal models of colitis.

In conclusion, the present study demonstrated the expression of IRAK-3 and gene methylation of IRAK-3 in the colons of mice with chemically-induced UC. The results revealed that DSS and DMH induced the downregulation of IRAK-3 and methylation of a region of IRAK-3, increased inflammation in the colon, and increased the risk of tumorigenesis. Together, these findings suggested that IRAK-3 gene methylation may be a predictive factor in the transition from colitis to cancer, and IRAK-3 may be a potential therapeutic target for colitis and tumorigenesis.

\section{Acknowledgements}

The present study was supported by the Science and Technology Project of Zhejiang Province, China (grant no. 2012C37105) and the Natural Science Foundation of Zhejiang Province, China (grant no. LY15H160040).

\section{References}

1. Danese S and Fiocchi C: Ulcerative colitis. N Engl J Med 365: 1713-1725, 2011.

2. Low D, Nguyen DD and Mizoguchi E: Animal models of ulcerative colitis and their application in drug research. Drug Des Devel Ther 7: 1341-1357, 2013.

3. Mombaerts P, Mizoguchi E, Grusby MJ, Glimcher LH, Bhan AK and Tonegawa S: Spontaneous development of inflammatory bowel disease in T cell receptor mutant mice. Cell 75: 274-282, 1993.

4. Eaden JA, Abrams KR and Mayberry JF: The risk of colorectal cancer in ulcerative colitis: A meta-analysis. Gut 48: 526-535, 2001.

5. Fukata M, Shang L, Santaolalla R, Sotolongo J, Pastorini C, España C, Ungaro R, Harpaz N, Cooper HS, Elson G, et al: Constitutive activation of epithelial TLR4 augments inflammatory responses to mucosal injury and drives colitis-associated tumorigenesis. Inflamm Bowel Dis 17: 1464-1473, 2011.

6. Biswas A, Wilmanski J, Forsman H, Hrncir T, Hao L, Tlaskalova-Hogenova $\mathrm{H}$ and Kobayashi KS: Negative regulation of Toll-like receptor signaling plays an essential role in homeostasis of the intestine. Eur J Immunol 41: 182-194, 2011.

7. Jain A, Kaczanowska S and Davila E: IL-1 receptor-associated kinase signaling and its role in inflammation, cancer progression, and therapy resistance. Front Immunol 5: 553, 2014.

8. Berglund M, Melgar S, Kobayashi KS, Flavell RA, Hörnquist EH and Hultgren $\mathrm{OH}$ : IL-1 receptor-associated kinase M downregulates DSS-induced colitis. Inflamm Bowel Dis 16: 1778-1786, 2010.

9. Zhou H, Yu M, Fukuda K, Im J, Yao P, Cui W, Bulek K, Zepp J, Wan Y, Kim TW, et al: IRAK-M mediates Toll-like receptor/IL-1R-induced NFKB activation and cytokine production. EMBO J 32: 583-596, 2013.
10. Kobayashi K, Hernandez LD, Galán JE, Janeway CA Jr, Medzhitov R and Flavell RA: IRAK-M is a negative regulator of Toll-like receptor signaling. Cell 110: 191-202, 2002.

11. Ullman TA and Itzkowitz SH: Intestinal inflammation and cancer. Gastroenterology 140: 1807-1816, 2011.

12. Livak KJ and Schmittgen TD: Analysis of relative gene expression data using real-time quantitative PCR and the 2(-Delta Delta C(T)) Method. Methods 25: 402-408, 2001.

13. Tanaka T, Kohno H, Suzuki R, Yamada Y, Sugie S and Mori H: A novel inflammation-related mouse colon carcinogenesis model induced by azoxymethane and dextran sodium sulfate. Cancer Sci 94: 965-973, 2003.

14. Okayasu I, Hatakeyama S, Yamada M, Ohkusa T, Inagaki Y and Nakaya R: A novel method in the induction of reliable experimental acute and chronic ulcerative colitis in mice. Gastroenterology 98: 694-702, 1990.

15. Chen W, Saxena A, Li N, Sun J, Gupta A, Lee DW, Tian Q, Dobaczewski M and Frangogiannis NG: Endogenous IRAK-M attenuates postinfarction remodeling through effects on macrophages and fibroblasts. Arterioscler Thromb Vasc Biol 32: 2598-2608, 2012

16. Hanahan D and Weinberg RA: Hallmarks of cancer: The next generation. Cell 144: 646-674, 2011.

17. Klimesova K, Kverka M, Zakostelska Z, Hudcovic T, Hrncir T, Stepankova R, Rossmann P, Ridl J, Kostovcik M, Mrazek J, et al: Altered gut microbiota promotes colitis-associated cancer in IL-1 receptor-associated kinase M-deficient mice. Inflamm Bowel Dis 19: 1266-1277, 2013.

18. del Fresno C, Otero K, Gómez-García L, González-León MC, Soler-Ranger L, Fuentes-Prior P, Escoll P, Baos R, Caveda L, García F, et al: Tumor cells deactivate human monocytes by up-regulating IL-1 receptor associated kinase-M expression via CD44 and TLR4. J Immunol 174: 3032-3040, 2005.

19. Soares-Schanoski A, Jurado T, Córdoba R, Siliceo M, Fresno CD, Gómez-Piña V, Toledano V, Vallejo-Cremades MT, Alfonso-Iñiguez S, Carballo-Palos A, et al: Impaired antigen presentation and potent phagocytic activity identifying tumor-tolerant human monocytes. Biochem Biophys Res Commun 423: 331-337, 2012.

20. Standiford TJ, Kuick R, Bhan U, Chen J, Newstead M and Keshamouni VG: TGF- $\beta$-induced IRAK-M expression in tumor-associated macrophages regulates lung tumor growth. Oncogene 30: 2475-2484, 2011.

21. Wheeler JM, Kim HC, Efstathiou JA, Ilyas M, Mortensen NJ and Bodmer WF: Hypermethylation of the promoter region of the E-cadherin gene (CDH1) in sporadic and ulcerative colitis associated colorectal cancer. Gut 48: 367-371, 2001.

22. Sato F, Shibata D, Harpaz N, Xu Y, Yin J, Mori Y, Wang S, Olaru A, Deacu E, Selaru FM, et al: Aberrant methylation of the HPP1 gene in ulcerative colitis-associated colorectal carcinoma. Cancer Res 62: 6820-6822, 2002.

23. Kang GH, Lee HJ, Hwang KS, Lee S, Kim JH and Kim JS: Aberrant $\mathrm{CpG}$ island hypermethylation of chronic gastritis, in relation to aging, gender, intestinal metaplasia, and chronic inflammation. Am J Pathol 163: 1551-1556, 2003.

24. Jang TJ, Kim DI, Shin YM, Chang HK and Yang CH: p16(INK4a) Promoter hypermethylation of non-tumorous tissue adjacent to gastric cancer is correlated with glandular atrophy and chronic inflammation. Int J Cancer 93: 629-634, 2001.

25. Saito S, Kato J, Hiraoka S, Horii J, Suzuki H, Higashi R, Kaji E, Kondo Y and Yamamoto K: DNA methylation of colon mucosa in ulcerative colitis patients: Correlation with inflammatory status. Inflamm Bowel Dis 17: 1955-1965, 2011. 